\title{
Longitudinal bunch profile diagnostic for magnetized electron beams
}

\author{
Mark Stefani® ${ }^{1}$ and Fay Hannon ${ }^{2}$ \\ ${ }^{1}$ Old Dominion University, Department of Electrical Engineering, Norfolk, Virginia 23529, USA \\ ${ }^{2}$ Thomas Jefferson National Accelerator Facility Newport News, Virginia 23606, USA
}

(Received 21 February 2019; published 8 November 2019)

\begin{abstract}
The study of magnetized electron beam has become a high priority for its use in ion beam cooling as part of electron ion colliders and the potential of easily forming flat beams with a large aspect ratio. In this paper, a new diagnostic is described with the purpose of studying longitudinal and transverse magnetized beam properties. The device is a modification to a typical pepper-pot. Specifically, this 1D pepper-pot was designed for use with a transverse deflecting cavity for longitudinal bunch profile measurements of magnetized beams.
\end{abstract}

DOI: 10.1103/PhysRevAccelBeams.22.112802

\section{INTRODUCTION}

A magnetized electron beam is formed when emitted electrons from the cathode are exposed to a magnetic field perpendicular to the cathode surface; a condition that can be created by placing the cathode in a uniformed region of field produced by a solenoid. As the electrons exit the magnetic fringe field, they acquire angular momentum through the conservation of momentum. The average angular canonical momentum scales with the peak longitudinal field on the cathode and the emitting radius squared. Magnetized beams have been demonstrated primarily for the formation of flat electron beams that have extreme transverse aspect ratios [1,2]. For highly magnetized bunches, the transverse beam size increases rapidly during drift due to the contribution of canonical angular momentum to the transverse emittance. This inherent angular momentum of magnetized beams manifests as a rotation and natural divergence in the transverse plane that complicates traditional diagnostic techniques, as will be described in the following sections.

Research at Thomas Jefferson National Accelerator Facility (JLab) has focused on the production of magnetized electron beams $[3,4]$ for the purpose of an electron cooler for the Jefferson Laboratory Electron Ion Collider (JLEIC) [5,6]. It was proposed that the cooling rate between a copropagating electron and ion beam in a solenoid channel could be improved by about two orders of magnitude if the electron bunch was not following standard Larmor rotations [7-9]. This would be the case if the angular momentum from a

Published by the American Physical Society under the terms of the Creative Commons Attribution 4.0 International license. Further distribution of this work must maintain attribution to the author(s) and the published article's title, journal citation, and DOI. magnetized beam could be removed through the fringe fields of the cooling channel solenoid.

The design criteria for the injector for the JLEIC cooler requires $3.2 \mathrm{nC}$ per electron bunch at $43.3 \mathrm{MHz}$ repetition rate [5]. To produce both the bunch charge and average current, a thermionic gun is being considered. A demonstration of this gun technology as a magnetized source is being constructed at JLab with a short diagnostic beam-line that will be used to characterize the electron beam as a function of magnetization, as shown in Fig. 1. The thermionic gun to be tested will operate at $500 \mathrm{MHz}$ with a nominal bunch charge of $130 \mathrm{pC}$ an energy of $125 \mathrm{keV}$ and initial bunch length of $210 \mathrm{ps}$. The longitudinal measurement will utilize a 1D pepper-pot located at $1.98 \mathrm{~m}$, a deflecting cavity at $2.68 \mathrm{~m}$, and a YAG screen viewer at $3.4 \mathrm{~m}$.

\section{LONGITUDINAL BUNCH PROFILE DIAGNOSTIC}

A longitudinal measurement via a deflecting-mode cavity is achieved using a rf cavity to provide a zero-crossing-angle deflection of a bunch, which causes a longitudinal crabbing effect. The transverse profile is then viewed on a screen and a calculation can extract the bunch length. By scaling the intensity distribution observed on the new transverse projection to match the calculated bunch length, an accurate longitudinal profile measurement is created $[10,11]$. Smaller transverse beams in the deflecting plane, that are not dominated by space charge forces, improve the accuracy of the measurement. It can therefore be advantageous to pass the beam through a slit aperture, producing a nonspace charge dominated beamlet, as it removes inherent errors within the measurement to be discussed further in Sec. II B. Even with a slit aperture it is more problematic to interpret the image of a magnetized beam from a viewer. This is because the angular momentum is still present, causing the individual beamlets to rotate transversely. 


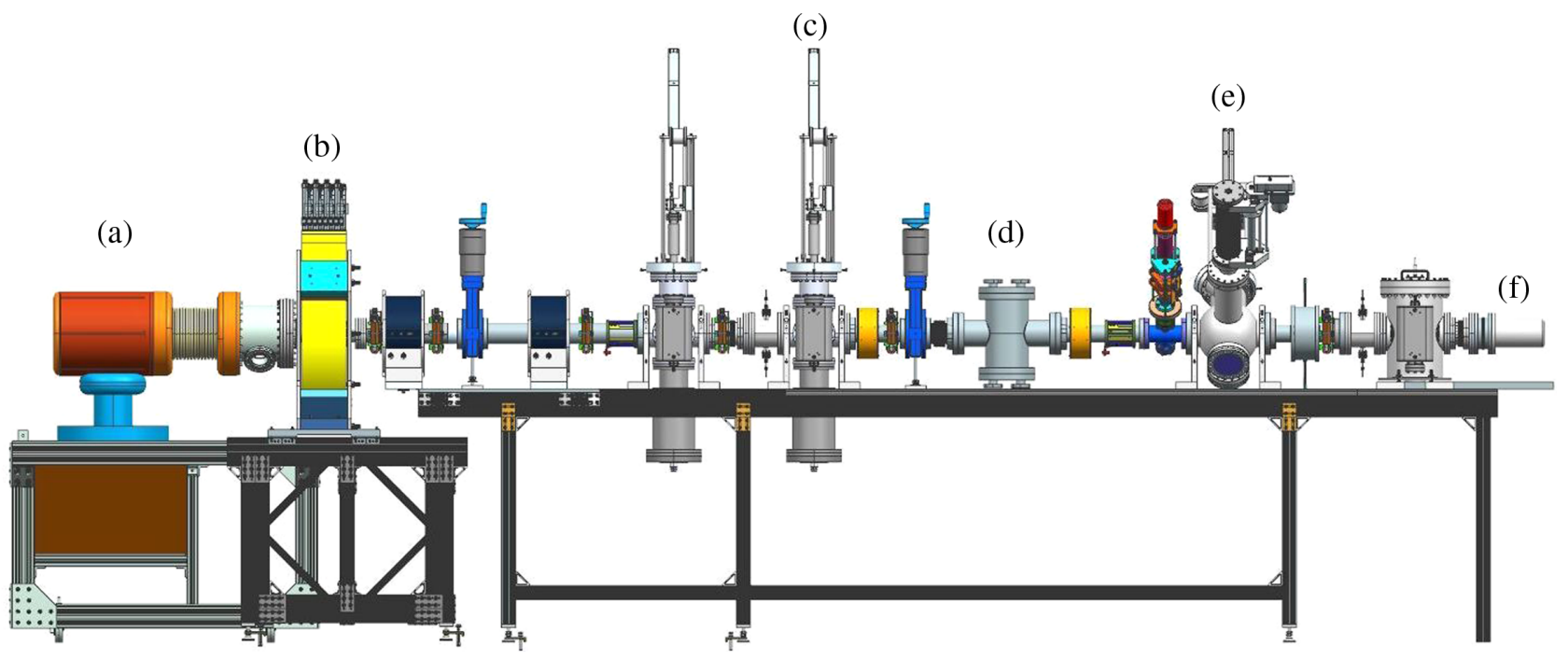

FIG. 1. Mechanical model of the test beamline (a) thermionic gun (b) mgnetizing solenoid (c) housing for 1D pepper-pot (d) deflecting cavity (e) housing for viewer screen (f) beam dump.

In general, with magnetized bunches, the dynamics of the beam becomes increasingly challenging to incorporate for accurate analysis of measurements taken. However, a combination of a slit and pepper-pot mitigates these issues. Removing multiple rows of a traditional 2D pepper-pot, keeping a single array of holes centered in the beamline, one maintains the benefits of the slit; while having the ability to distinguish beamlets.

By combining these two typical devices, one loses no functionality of either diagnostic when beam scanning is incorporated. Knowing the final positions of the individual beamlets with respect to the initial positions of the holes on the $1 \mathrm{D}$ pepper-pot, one can make measurements related to angular momentum and transverse emittance using common techniques. The procedures related to measurements with this device is no different from the conditions and process one would use with a traditional slit [12], as discussed in Sec. III.

\section{A. 1D pepper-pot design}

The design for the array of holes had three primary conditions to meet. First, the beamlets must be distinct from each other even after a drift and transverse deflection. Second, the beamlets must be identifiable to their initial location. Third, the percentage of the beam passing through the 1D pepper-pot should ensure consistent operation over a range of required average currents (transverse beam size varies with current and the magnetizing field strength). By considering these three conditions along with confirmation of their application via simulation, values for design of the 1D pepper-pot were finalized.

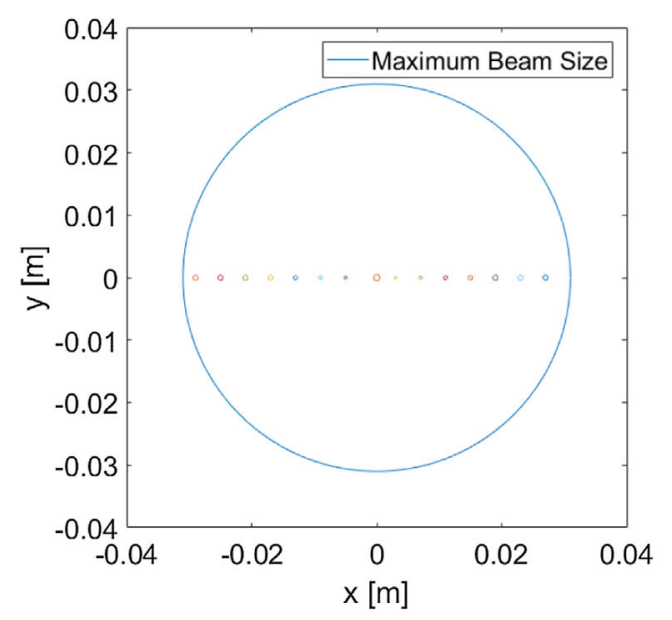

(a)

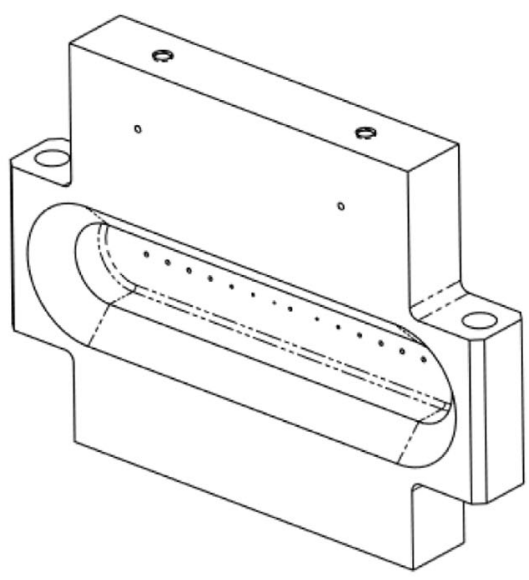

(b)

FIG. 2. (a) Schematic of relative hole sizes and location (b) Mechanical drawing of the device. 


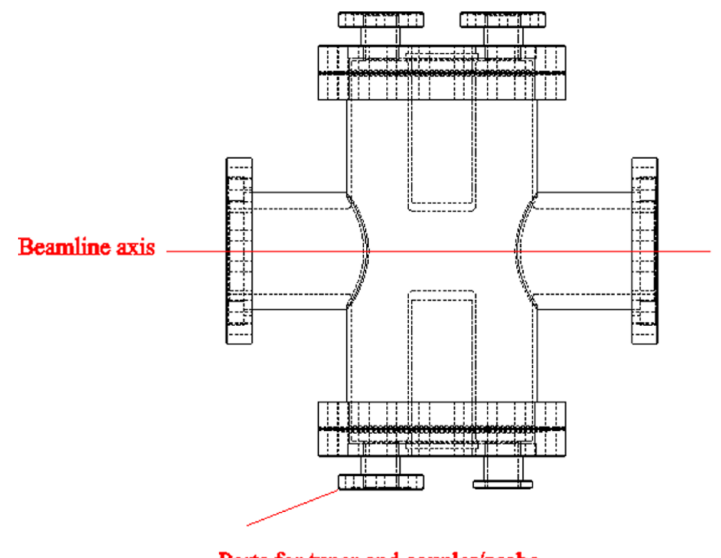

Ports for tuner and coupler/probe

(a)

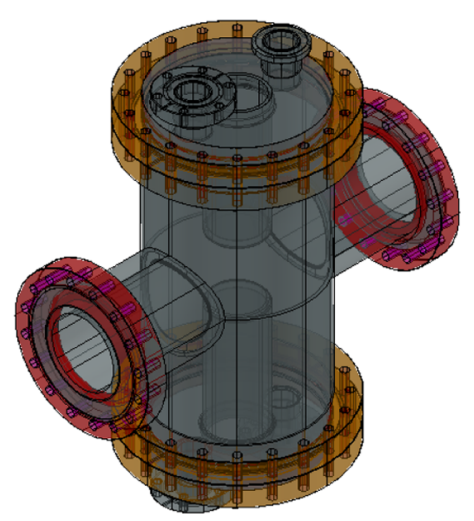

(b)

FIG. 3. (a) Schematic of DQWR cavity with beam axis and ports indicated. (b) Transparent 3D drawing of DQWR cavity.

The design calls for a single horizontal array of holes of varying sizes across the diameter of the beam pipe on a tungsten-copper alloy plate, chosen for heat conductance. Figure 2 shows schematically the hole layout. The distribution and various sizes of these holes were chosen as a result of beam dynamics simulations over the expected parameter space. Hole sizes were selected to allow a roughly similar percentage of beam to pass through for varying beam sizes. In order to accomplish this, the holes must increase in size as their displacement from the center increases (range from $300 \mu \mathrm{m}$ to $650 \mu \mathrm{m}$ ). The spacing was chosen so beamlets are distinguishable from one another after a drift to the location of the viewer. In order to determine the exact starting position of each beamlet, an off-set was given to the right side of the array and then a noticeable difference in hole size between the center and closest hole was made to ensure they do not overlap after the drift. A bevel and counter sink were used to avoid

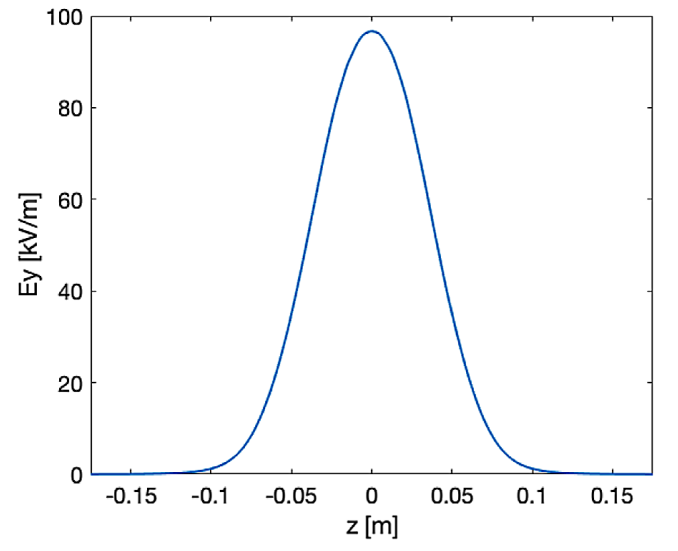

\begin{tabular}{lll}
\hline Parameters & Unit & Value \\
\hline Frequency $f$ & $\mathrm{MHz}$ & 500 \\
RF coupling $\beta$ & - & 1 \\
Shunt impedance & $\mathrm{M} \Omega$ & 4.43 \\
Quality factor $Q_{0}$ & - & 10722 \\
\hline
\end{tabular}

(b) Simulated figures of merit of the DQWR.

(a) The profile along the beam axis of electric field producing vertical deflection in the DQWR

scraping of the beamlets after passing through the hole and for ease of manufacture.

\section{B. Double quarter wave resonator cavity design}

The design of the double quarter wave resonator (DQWR) cavity for use in longitudinal measurements is shown in Fig. 3. All items are brazed, except the joints joining the beam-pipe and cavity body, which is welded using an electron beam.

The deflection in the DQWR is from the Lorentz force provided by a TEM (fundamental) mode, whose main contribution comes from the electric field rather than the magnetic field. With $\mathrm{z}$ and $\mathrm{y}$ axes aligned with the beam axis and the vertical axis of the DQWR respectively, the force in the direction of deflection (y axis) is given as

$$
F_{y}=e\left(E_{y}+v_{z} B_{x}\right)
$$

FIG. 4. DQWR details. 
where $e$ is electron charge and $v_{z}$ is the velocity of electron along the beam axis. The 1D E-field profile on the beam axis is shown in Fig. 4.

The design and field maps for this cavity were created using computer simulation technology (CST) software [13]. The exported fields where then used in simulations in the space charge tracking code GPT [14]. Values from both CST and GPT are used to calculated operating voltage and power requirements.

The raw fields are exported from CST as a 3D $\operatorname{Re}[E]$ and $\operatorname{Im}[H]$ field. CST by default creates fields based on 1 Joule of energy. In the GPT simulation a scaling factor is then applied to these fields to give the required deflection to the bunch. $H$ is converted to $B$ by the factor $\mu_{0}$ and the only values extracted from GPT is longitudinal beta and the scaling factor. The wave number $\mathrm{k}$ for the rf is given by $\frac{2 \pi}{\lambda}$. These values are all used in the calculation for the deflecting voltage applied to the bunch [15].

$$
V_{\perp}=\int_{-\infty}^{\infty} \frac{E_{\perp}}{\beta} e^{\frac{i k z}{\beta}}-i c B_{\perp} e^{\frac{i k z}{\beta}} d z
$$

$k \approx 10.45, E_{\perp}=E_{y}(z), B_{\perp}=B_{x}(z)$, longitudinal beta: $\beta=0.595$, scaling factor from simulation: $S=0.007$

$$
V_{\perp}=1.36 \mathrm{MV}
$$

Therefore, applying the scaling factor, the operating deflecting voltage is $9.5 \mathrm{kV}$.

The required power is proportional to the field squared. Therefore, the power loss calculated in CST as $289 \mathrm{~kW}$ times the scaling factor squared gives the lower bounds for power required to operate the cavity as $14.1 \mathrm{~W}$; not including dissipated power. However, the measured Q of the cavity is roughly $20 \%$ below CST simulation. Which means the power can be expected to be increased by $20 \%$ giving a new lower bound of $16.92 \mathrm{~W}$. The lower power of the cavity is not surprising, given the low energy of the beam that is being deflected. The lower power of the cavity is not surprising, given the low energy of the beam that is being deflected.

\section{LONGITUDINAL BUNCH PROFILE: MEASUREMENT ERROR, CALCULATION, AND SIMULATION}

The advantage of this new method utilizing the 1D pepper-pot is that it removes an error in the standard procedure for longitudinal measurements. Specifically, the error that results from overestimation of bunch length because of contributions to the measurement from transverse size. In general, a bunch being deflected will not give a purely longitudinal projection, as shown in Fig. 5. Without an ideal projection, the initial transverse size of the bunch causes an inaccuracy to the image, because the projected view will be the length of the tilted bunch from the top of the previously normal transverse face to the bottom of the previously transverse face; when the correct length will be the distance from the center of the initially transverse face in the front to the center of the transverse face in the back of the bunch. The error arises from not knowing precisely how much the initially transverse face of the bunch is contributing to the projected longitudinal size.

However, by passing the beam through a 1D pepper-pot a small beamlet can be deflected to give a much more accurate measurement as shown in the following Fig. 6.

Furthermore, large bunch sizes are limited in possible longitudinal projection, either due to size of viewer or the beam pipe. With a beamlet a much larger transverse deflection can be applied and therefore we get a more accurate longitudinal projection. This comparison is clear from simulation in Figs. 7 and 8 for magnetized beam.

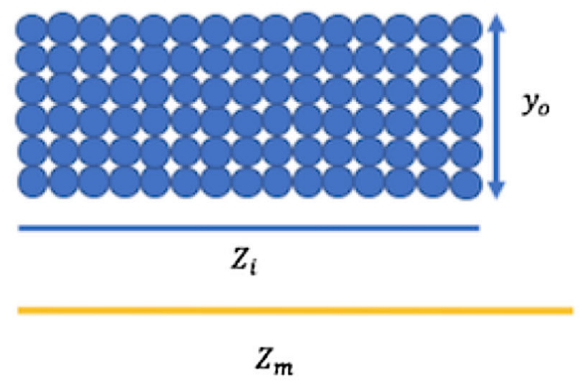

(a)

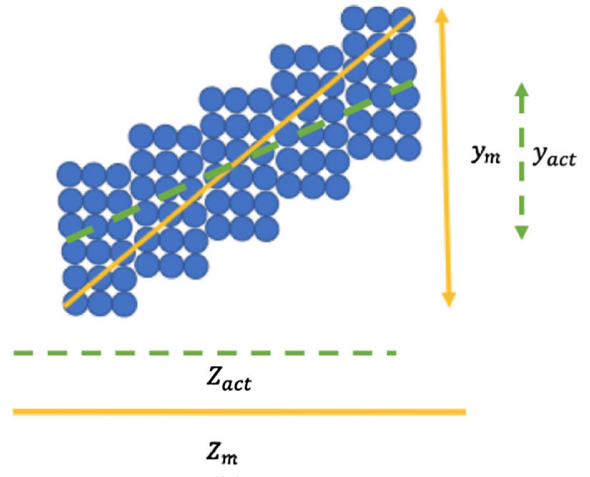

(b)

FIG. 5. (a) Initial longitudinal view of idealized bunch from the side. $y_{0}$ is the transverse size without deflection, $Z_{i}$ is the initial longitudinal bunch length (b) Longitudinal view of a deflected idealized bunch from the side. $y_{m}$ is the measured transverse projection after deflection, $y_{\text {act }}$ is the value that would ideally be measured, $Z_{m}$ will be the calculated bunch length from the measurement, $Z_{\text {act }}$ is the ideally calculated length. Visual inspection comparing $Z_{i}$ and $Z_{\text {act }}$ to $Z_{m}$ demonstrates the concept of how this error overestimates the true bunch length. 


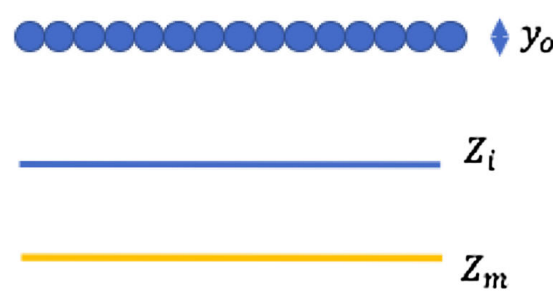

(a)

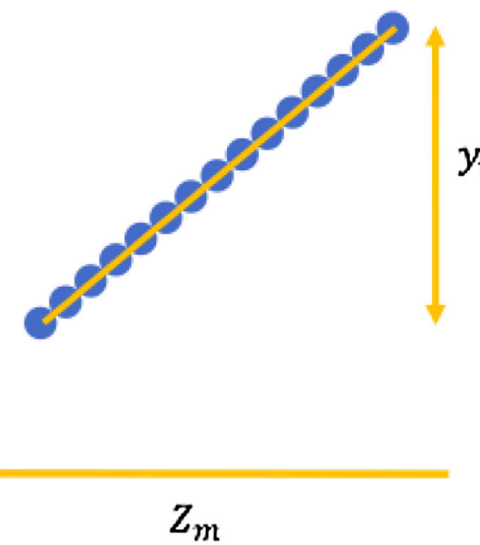

(b)

FIG. 6. (a) Initial longitudinal view of one idealized beamlet after the 1D pepper-pot. $y_{0}$ is the transverse size without deflection, $Z_{i}$ is the initial longitudinal bunch length. (b) Longitudinal view of a deflected idealized beamlet and projected size at viewer. $y_{m}$ is the measured transverse projection after deflection, $Z_{m}$ will be the calculated bunch length from the measurement. Visual inspection comparing $Z_{i}$ and $Z_{m}$ shows the true bunch length can be recovered by removing the contribution of the transverse face of the bunch to the projected size.

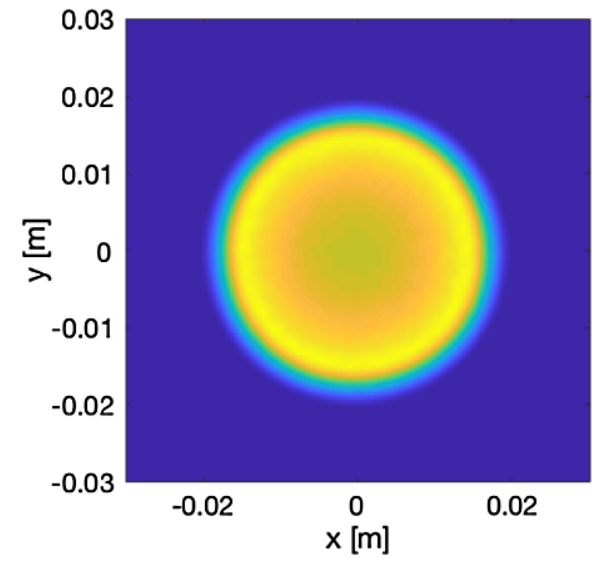

(a)

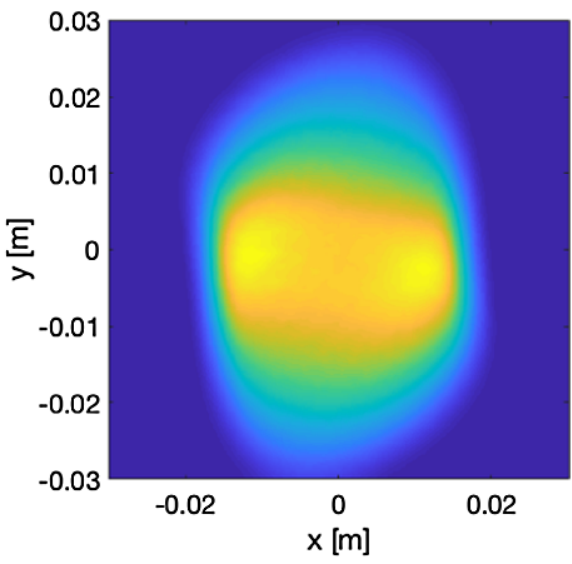

(b)

FIG. 7. (a) Transverse view of bunch without deflection, (b) transverse view of a deflected bunch at viewer.

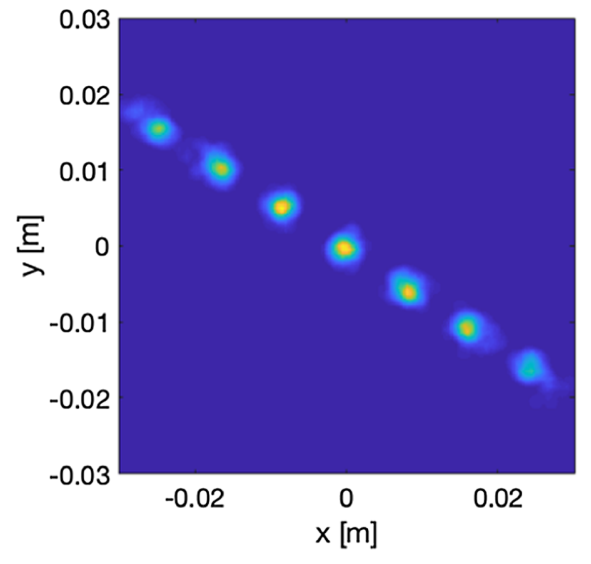

(a)

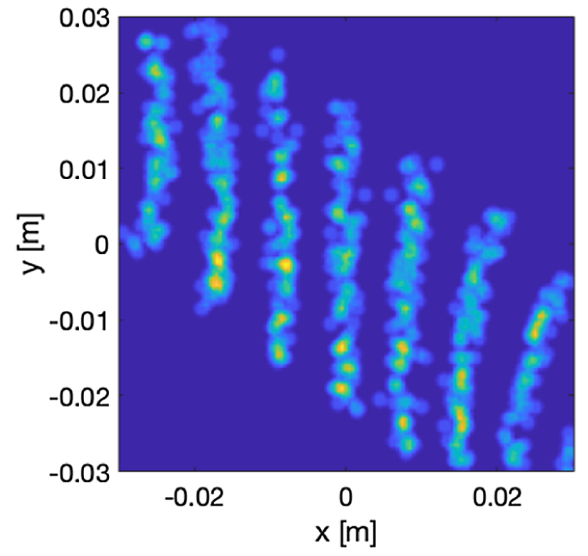

(b)

FIG. 8. (a) Transverse view of beamlets without deflection, (b) transverse view of all deflected beamlets at viewer. 
The analysis to calculate the bunch length utilizes Eq. (3) $[7,8]$ :

$$
\sigma_{y}=\sqrt{\sigma_{y 0}^{2}+\sigma_{z}^{2} \beta_{a} \beta_{s}\left[\frac{2 \pi e V_{0}}{\lambda p c} \sin (\Delta \Psi) \cos (\phi)\right]^{2}},
$$

where $\sigma_{y}$ is the measured transverse size after the deflection, $\sigma_{y 0}$ is the initial transverse bunch size. Or in the case of the mask, the hole diameter. $\sigma_{z}$ is the unknown bunch length, $V_{0}$ is the voltage used from the transverse deflection, $\lambda$ is the wavelength of the transverse deflecting field which will match the length associated with the frequency from the electron source, $p$ is the momentum and $c$ is the speed of light, $e$ is the fundamental charge, $\beta_{a}$ is the Courant-Snyder beta of the bunch at the location of deflection and $\beta_{s}$ is the Courant-Snyder beta of the bunch at the viewer. The geometric mean of these values is close to the drift length from the transfer matrix, $D . \Delta \Psi$ is the betatron phase advance and is taken to be $\frac{\pi}{2}$. Finally, $\phi$ is the crossing angle and should be 0 for the best measurement. Applying the conditions and solving for bunch length leads to the following calculation, Eq. (4).

$$
\begin{aligned}
p c & =\sqrt{K E^{2}+2 K E m_{o} c^{2}}, \sqrt{\beta_{a} \beta_{s}} \approx R_{12}=D, \\
\Delta \Psi & =\frac{\pi}{2}, \phi=0 \\
\sigma_{z} & =\frac{\lambda \sqrt{\left(K E^{2}+2 K E m_{o} c^{2}\right)\left(\sigma_{y}^{2}-\sigma_{y 0}^{2}\right)}}{2 \pi e V_{0} D} .
\end{aligned}
$$

This value is used to scale the distribution measured by the viewer and reproduces an accurate longitudinal profile of the bunch.

\section{A. Simulated example}

A virtual experiment was simulated for this diagnostic, performed using GPT. 100k particles were used for a $130 \mathrm{pC}$ bunch at $125 \mathrm{keV}$ energy with a $8 \mathrm{~mm}$ diameter initial transverse size and initial truncated cosine longitudinal

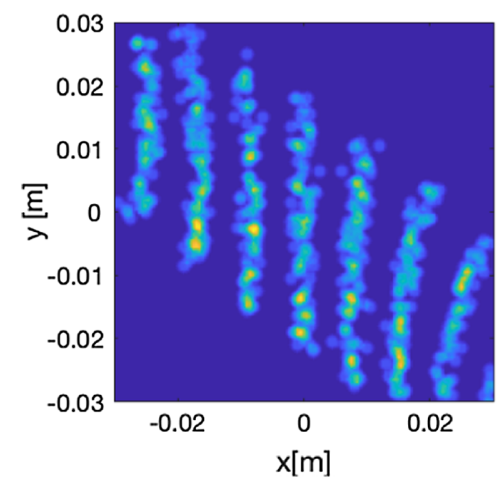

(a) profile as predicted by gridded thermionic gun operation. A magnetic field of $306.8 \mathrm{G}$ at the cathode surface was used to create the desired magnetization. The particle distribution was tracked from the cathode to the location of the 1D pepper-pot including space charge forces and the effects of all beam-line elements such as solenoids. The solenoids were used to maintain transverse size to within the beampipe diameter and to have the beam at waist when reaching the $1 \mathrm{D}$ pepper-pot to accommodate the long drift to the viewer. No steering was necessary in the simulation, but in reality, correctors must be used to counteract the effect of the earths magnetic field even in an ideal case.

A 1D pepper-pot mask was applied with hole sizes $(0.8 \mathrm{~mm})$ that passed enough particles (in total, roughly $1 \%$ ) through the mask, such that a profile could be fitted to the simulated streak at the viewer location $1.42 \mathrm{~m}$ away. The beamlets were tracked through simulated deflecting cavity's rf field, producing the required transverse deflection and longitudinal projection. The deflected beamlets are then tracked to the viewer location. The simulated viewer image is shown in Fig. 9(a). The image in Fig. 9(a) appears speckled due to some regions of the deflected bunch being defuse in terms of particles density relative to other portions. This stems from the simulation being performed with just enough particles to allow for accurate reconstruction while maintaining a realistically limiting aperture.

In post processing, before the bunch profile reconstruction, the average rotation of the simulated viewer image was removed and a density profile is made along the vertical axis of all the beamlets, as shown in Fig. 9(b). This same profile is then scaled using details from Eq. (4) and is then fitted to a cosine curve via least-squares function approximation, which yields Fig. 10. The function is fitted to a cosine based on gun operation, as it is known that the bunch profile should approximate a truncated cosine. The bunch length measurement is taken as the full-width-halfmaximum (FWHM), producing a value of 232 ps. This corresponds to covering $41^{\circ}$ of the full waveform from the gun's operating frequency $(500 \mathrm{MHz}) .41^{\circ}$ is still within a

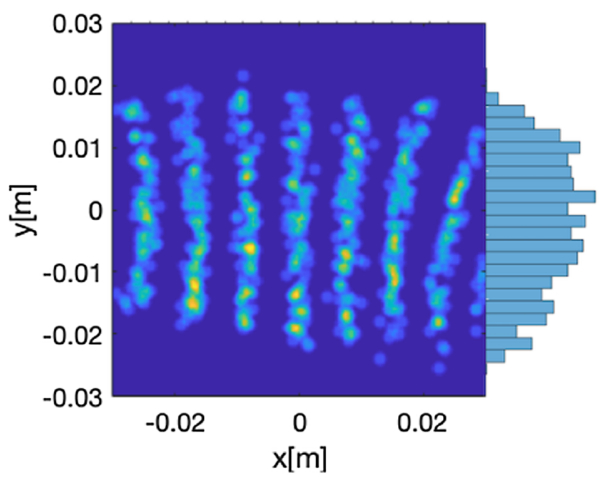

(b)

FIG. 9. (a) Simulated viewer image of deflected beamlets from 1D pepper-pot, (b) Image with rotation removed and histogram profile prescaling. 


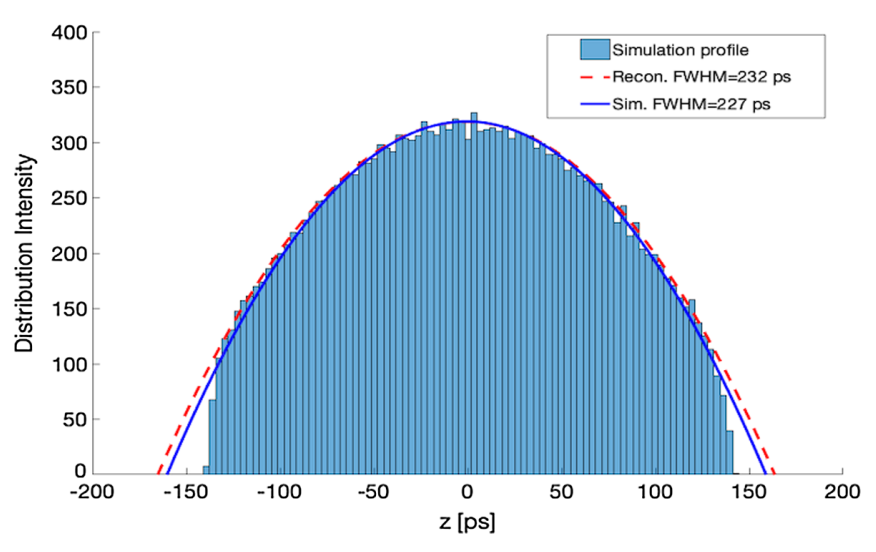

FIG. 10. Directly simulated and reconstructed longitudinal bunch profile at location of $1 \mathrm{D}$ pepper-pot.

linear regime which indicated that the equation for scaling and function fitting are valid. 232 ps compares well to the FWHM of 227 ps from the cosine fitted to the true distribution at the location of the 1D pepper-pot.

\section{CONCLUSION}

As the study of magnetized electron beam continues to be a high priority for its multitude of uses, this new diagnostic for studying longitudinal and transverse magnetized beam properties shows promise for characterization of these magnetized $e^{-}$beams. Normally, in the process of making a longitudinal measurement, the deflected bunch will not give a purely longitudinal projection causing an inaccuracy to the projected image and an overestimate of the bunch size. An aperture limiting the transverse bunch size helps remove this error. This is because a more accurate measurement can be made with a smaller beam in the deflecting plane. Holes help to determine where to calculate the longitudinal profile $f(z)$ which is difficult otherwise because of the shear due to magnetization. Moreover, if the rotation from magnetization is not linear, it is still possible to make a fit to the rotation. By simply changing the slit into individual holes, each beamlet can be tracked back to its initial location allowing for an accurate longitudinal profile along the whole transverse face of the bunch which provides $f(z)$ vs $x$ instantly. This implies that if the beam is scanned in y, one can get $f(z)$ vs $y$ which is not possible with a slit or $2 \mathrm{D}$ pepper pot.

This novel technique using a new design of a 1D pepper-pot has shown through simulation its usefulness and accuracy in measurements of longitudinal bunch profile of magnetized beams when used with a deflecting cavity. Removing the errors of the measurement and having well defined terms to calculated the bunch length the simulated measurement was able to reproduce a bunch profile with an error of $2.16 \%$ from the known bunch FWHM. This ideal simulation demonstrates selfconsistency, to good accuracy, utilizing the 1D pepper-pot for longitudinal measurements.

\section{ACKNOWLEDGMENTS}

This manuscript has been authored by Jefferson Science Associates, LLC under U.S. DOE Contract No. DE-AC05-06OR23177. The publisher, by accepting the article for publication, acknowledges that the United States Government retains a non- exclusive, paid-up, irrevocable, world-wide license to publish or reproduce the published form of this manuscript, or allow others to do so, for United States Government purposes. Xelera Research LLC was funded by the U.S. DOE's Office of Nuclear Physics SBIR Program No. DE-SC0009516. M. Stefani is currently conducting research at Jefferson Lab toward a Ph.D. degree.

[1] P. Piot Y.-E Sun, and K.-J. Kim, Photoinjector generation of a flat electron beam with transverse emittance ratio of 100, Phys. Rev. Accl. Beams 9, 031001 (2006).

[2] Y. Sun and P. Piot, Summary of the angular-momentumdominated beam experiment, University of Chicago, Fermi Lab, 2004.

[3] M. A. Mamun et al., Production of magnetized electron beam from a dc high voltage photogun, in IPAC'18, http://dx .doi.org/10.18429/JACoW-IPAC2018-THPMK108 (2018).

[4] S. A. K. Wijethunga et al., Simulation study of the magnetized electron beam, in IPAC'18, http://dx.doi.org/10 .18429/JACoW-IPAC2018-THPAK071 (2018).

[5] H. Zhang, Y. Zhang et al., Multi-stage electron cooling scheme for JLEIC, in IPAC'18, http://dx.doi.org/10.18429/ JACoW-IPAC2018-MOPML006 (2018).

[6] Stephen Benson et al., Development of bunched beam electron cooler for The Jefferson Lab electron-ion collider, in IPAC18, http://dx.doi.org/10.18429/JACoW-IPAC2018MOPMK015 (2018).

[7] Y.E. Sun and P. Piot, Generation and dynamics of magnetized electron beams for high-energy electron cooling, Report No. FERMILAB-CONF-14-142-APC, 2014.

[8] Ya. Derbenev, University of Michigan Report No. UM-HE98-04, 1998.

[9] A. S Artamonov and Ya. Derbenev, Positron cooling in a magnetized electron beam, Zh. Eksp. Teor. Fiz. 94, 15 (1988).

[10] P. Emma et al., A transverse rf deflecting structure for bunch length and phase space diagnostics, SLAC, Report No. LCLS-TN-00-12, 2000.

[11] R. Akre et al., Bunch Length Measurement Using A Transverse RF Deflecting Structure in the SLAC LINAC (SLAC, Stanford, CA, 2002).

[12] F. Hannon and M. Stefani, Transverse uncorrelated emittance diagnostic for magnetized electron beams, Phys. Rev. Accel. Beams 22, 102801 (2019).

[13] http://www.cst.com.

[14] S. B van der Geer and M. J de Loos, General Particle Tracer User Manual, Version 3.35, http://projects.astec.ac.uk/ EBTFManual/images/4/42/UserManual.pdf.

[15] D. Alesini, G. Di Pirro, L. Ficcadenti, A. Mostacci, L. Palumbo, J. Rosenzweig, and C. Vaccarezza, Rf deflector design and measurements for the longitudinal and transverse phase space characterization at SPARC, Nucl. Instrum. Methods Phys. Res, Sect. A 568, 488 (2006). 